\title{
The molecular mechanism of luteolin-induced apoptosis is potentially related to inhibition of angiogenesis in human pancreatic carcinoma cells
}

\author{
XUETING CAI ${ }^{1}$, WUGUANG LU ${ }^{1}$, TINGMEI YE ${ }^{2}$, MIN LU $^{3}$, JIANHUA WANG ${ }^{1}$, \\ JIEGE HUO $^{1}$, SHIHUI QIAN ${ }^{1}$, XIAONING WANG $^{1}$ and PENG CAO ${ }^{1}$

\begin{abstract}
${ }^{1}$ Laboratory of Cellular and Molecular Biology, Jiangsu Province Academy of Traditional Chinese Medicine, Nanjing 210028;
${ }^{2}$ Department of Biology, School of Chemistry and Life Sciences, Lishui University, Lishui 323000; ${ }^{3}$ State Key Laboratory of Pharmaceutical Biotechnology, School of Life Sciences, Nanjing University, Nanjing 210023, P.R. China
\end{abstract}

Received February 21, 2012; Accepted May 11, 2012

DOI: $10.3892 /$ or.2012.1914

\begin{abstract}
Luteolin has been shown to have a strong anticancer effect on various cancer models via programmed cell death (apoptosis). However, the fundamental mechanisms of these effects are still unclear. In the present study, we examined the question of whether or not luteolin can inhibit proliferation of pancreatic carcinoma cells, via apoptosis. We used three human pancreatic carcinoma cell lines, PANC-1, CoLo-357 and BxPC-3 in our study. In luteolin-treated pancreatic carcinoma cells, typical features of apoptosis were observed. Luteolin increased the expression of the pro-apoptotic protein Bax and decreased the expression of the anti-apoptotic protein Bcl-2, with a concomitant increase in the levels of caspase-3 and cleaved PARP after treatment for $24 \mathrm{~h}$. Luteolin inhibited HUVEC proliferation and vessel growth in CAM in vivo. In addition, the concentration of VEGF in the conditioned medium from human pancreatic carcinoma cells was downregulated by luteolin. Pancreatic carcinoma cells, pretreated with luteolin, could decrease the capillary-like structure formation by HUVEC, which was analyzed by a co-culture system. The abatement of VEGF secretion was related to the inhibition of VEGF mRNA expression, which may be regulated by inhibiting the transcription activity of nuclear transcription factor $\mathrm{NF}-\kappa \mathrm{B}$.
\end{abstract}

\section{Introduction}

The pancreas is the tenth most common site of new cancers, but pancreatic cancer is the fourth leading cause of cancer

Correspondence to: Dr Peng Cao, Laboratory of Cellular and Molecular Biology, Jiangsu Province Academy of Traditional Chinese Medicine, 100 Shizi Street, Hongshan Road, Nanjing 210028, Jiangsu, P.R. China

E-mail:pcao79@yahoo.com

Key words: luteolin, apoptosis, angiogenesis, VEGF, pancreatic carcinoma deaths among men and women, with an overall 5-year survival rate of $5 \%$ (1). One of the major causes of death is peritoneal dissemination and liver metastasis (2). The treatment of advanced pancreatic cancer with gemcitabine has only modest activity with a small survival benefit, and toxicity continues to be a major obstacle (3). New therapeutic strategies that notably lack cross resistance with established treatment regimens are much needed in pancreatic cancer. Since pancreatic carcinoma show strong tumor angiogenesis, overexpression of VEGF, the inhibition of tumor angiogenesis has been one of the promising strategies in the treatment of pancreatic carcinoma.

Luteolin, 3',4',5,7-tetrahydroxyflavone, is a common flavonoid that exists in many types of plants including fruits, vegetables and medicinal herbs. Plants rich in luteolin have been used in Chinese traditional medicine for treating various diseases such as hypertension, inflammatory disorders and cancer. The luteolin anticancer property is associated with the induction of apoptosis, and inhibition of cell proliferation, metastasis and angiogenesis (4). However, whether or not luteolin can inhibit proliferation of pancreatic carcinoma cells was unclear. In this study, we assessed the antitumor and antiangiogenic activity of luteolin on pancreatic carcinoma cells, and also investigated its effect on VEGF signal transduction.

\section{Materials and methods}

Materials. Luteolin was purchased from Nanjing TCM Institute of Chinese Materia Medica, China. Luteolin was dissolved in dimethyl sulfoxide (DMSO) and was used in all experiments. Trypsin, MTT [3-(4,5-dimethylthiazol2-yl)-2,5-diphenyltetrazolium bromide], propidium iodide (PI) and DNase-free RNase were obtained from Sigma, USA. Lysis buffer was purchased from Beyotime, China. Antibodies (caspase-3, caspase-8, goat anti-mouse IgG-HRP and goat anti-rabbit IgG-HRP) were obtained from Santa Cruz Biotechnology, CA, USA. Mouse anti-Bax was obtained from BD Biosciences, Bedford, MA, USA. Bcl-2, caspase-9, PARP (poly-ADP-ribose polymerase), ERK1/2, p-ERK1/2, p-p38, p38, JNK and p-JNK Antibody were purchased from Cell Signaling Technology, MA, USA. Monoclonal mouse 
anti-glyceraldehyde-3-phosphate dehydrogease (GAPDH) was obtained from KangChen, China. Endothelial cell growth supplement (ECGS) was purchased from Millipore, MA, USA. Peripheral blood was purchased from the Blood Center of Jiangsu Province, China. Ficoll-Hypaque was obtained from Meijing, China.

Cell culture. Human pancreatic carcinoma cell lines PANC-1, CoLo-357, BxPC-3 were purchased from CellBank of Shanghai Institute of Biochemistry and Cell Biology. Cells were cultured in DMEM-medium (for PANC-1, CoLo-357 cells) or RPMI-1640 (for BxPC-3 cells) supplemented with $10 \%$ fetal bovine serum (FBS), $100 \mathrm{U} / \mathrm{ml}$ penicillin and $100 \mu \mathrm{g} / \mathrm{ml}$ streptomycin (all available from Invitrogen, Grand Island, NY, USA). Human umbilical vein endothelial cells (HUVEC) were isolated from human umbilical cord veins by trypsin digestion method, improved from Jaffe et al (5). Fresh human umbilical cord was washed clear by phosphate-buffered saline (PBS), the vein was then infused with $0.1 \%$ trypsin and digested for $15 \mathrm{~min}$ at $37^{\circ} \mathrm{C}$. The cells digested from the umbilical vein was maintained in M199 medium supplemented with $15 \%$ FBS, $0.1 \mathrm{mg} / \mathrm{ml}$ heparin, $0.03 \mathrm{mg} / \mathrm{ml}$ endothelial cell growth supplement (ECGS). HUVEC were characterized by immunofluorescence method with antibodies to Factor VIII related antigen. Human peripheral blood mononuclear cells (PBMC) were isolated from fresh human blood by Ficoll-Hypaque density gradient centrifugation. The isolated cells were cultured in RPMI-1640 medium containing $10 \%$ FBS. All cultures were maintained in a humidified environment with $5 \% \mathrm{CO}_{2}$ at $37^{\circ} \mathrm{C}$.

Endothelial cell proliferation assay. HUVEC were seeded in 96-well plates, $1 \times 10^{4}$ cells per well. The medium was replaced by low-serum medium (1\% fetal bovine serum in $\mathrm{M} 199)$ containing different concentrations of luteolin. DMSO $(0.1 \%)$ was used as control. After $24 \mathrm{~h}$, the viability of HUVEC were analyzed by MTT assay as described previously (6). The percent inhibition of the treated cells was calculated by the formula: $\%$ inhibition $=1-\left(A_{570 \mathrm{~nm}}-A_{630 \mathrm{~nm}}\right)$ treated $/\left(A_{570 \mathrm{~nm}}-A_{630 \mathrm{~nm}}\right)$ control $\mathrm{x} 100 \%$. The $\mathrm{IC}_{50}$ were further assayed.

Cytotoxicity assay. The cytotoxicity of luteolin, which had potent HUVEC inhibition ability, was analyzed by MTT assay with PBMC and various differentiated human pancreatic carcinoma cell lines, the poorly differentiated cell line (PANC-1), the moderately differentiated cell line (CoLo-357) and the well differentiated cell line (BxPC-3).

Cell morphological assessment. Cells were cultured until mid-log phase. DMSO 0.1\% (control), 20, 40, 60 or $80 \mu \mathrm{mol} / 1$ luteolin was then added to the culture medium. The morphology of cells was monitored under an inverted microscope (Zeiss Axio Observer A1) at 6, 12, 18, 24 and $48 \mathrm{~h}$.

Cell cycle analysis. Cells treated with $0.1 \%$ DMSO or increasing concentrations of luteolin for different time periods were trypsinized and washed twice with PBS, and fixed in $100 \%$ ethanol overnight at $4^{\circ} \mathrm{C}$. Fixed cells were washed with PBS before incubation with $1 \mathrm{ml}$ PBS containing $50 \mu \mathrm{g} / \mathrm{ml} \mathrm{PI}$ and $1 \mathrm{mg} / \mathrm{ml} \mathrm{RNase}$ for $30 \mathrm{~min}$ at $37^{\circ} \mathrm{C}$. DNA content and cell cycle were determined using a FACScan laser flow cytometer (FACSCalibur, Becton-Dickinson, NY, USA). The data were analyzed using the software CellQuest.

Hoechst 33258 staining. Hoechst 33258 staining was used to visualize nuclear change and apoptotic body formation. At the end of luteolin treatment, attached cells were washed twice with PBS and fixed with $4 \%$ methanol at $4^{\circ} \mathrm{C}$ for $30 \mathrm{~min}$. The fixing solution was removed and cells were washed twice with PBS before staining with Hoechst 33258 (KeyGen, Nanjing, China). After staining for $10 \mathrm{~min}$, cells were washed again and observed under a fluorescence microscope (Zeiss Axio Observer A1) at $340 \mathrm{~nm}$.

Chicken chorioallantoic membrane (CAM) assay. Fertilized chicken eggs were incubated at around $55 \%$ relative humidity, $37.5^{\circ} \mathrm{C}$ incubator for 7 days. Eggs were set with the blunt end up at $45^{\circ} \mathrm{C}$ to prevent microbial growth and turned regularly (at least two times per day). At experiment day, eggs were checked with an egg candler, only live, fertilized ones were random divided, ten for each group. A $1.5 \times 1.5 \mathrm{~cm}$ 'window' was created on the blunt end of the egg, where the air sac was located. The air sac membrane was punctured carefully using an injection needle avoid breaking the blood vessel. About $50 \mu \mathrm{l}$ sterilized water was injected between the air sac membrane and chorioallantoic membrane, and then the air sac membrane could be easily peeled off. Sterilized filter paper $(5 \times 5 \mathrm{~mm})$ saturated with $0.1 \%$ DMSO (control) or luteolin (5 and $10 \mathrm{nmol} / \mathrm{egg}$ ) was air dried and then placed on the CAMs. The eggs were then covered with parafilm and put back to the incubator. Three days later, $20 \%$ fat emulsion (Chia-tai Tianqing Pharmaceutical Co., Jiangsu, China) was injected into the chorioallantois and the blood vessels were photographed by Sony $\alpha 100$ (Sony, Japan). Blood vessel density was calculated by Image-pro plus 6.0 software.

Tube formation assay. Under sterile conditions, 24-well plates were coated with $200 \mu \mathrm{l} /$ well of grow th factor-reduced Matrigel (BD Biosciences) without introducing air bubbles. The plates were set at $37^{\circ} \mathrm{C}$ for $30 \mathrm{~min}$ to allow gelling of Matrigel. The PANC-1 cells and HUVEC were cultured in serum-free medium for $12 \mathrm{~h}$ before the experiments. The HUVEC were then cultured on Matrigel, whereas the PANC-1 cells were pre-treated with $0.1 \% \mathrm{DMSO}$ or $40 \mu \mathrm{mol} / 1$ luteolin for $30 \mathrm{~min}$ before plating within the transwell inserts to prevent physical contact. Both cells were cultured in serum-free medium. The cells were co-cultured for $12 \mathrm{~h}$, HUVEC differentiated and formed capillary-like structures on Matrigel. The enclosed networks of complete tubes from five randomly chosen fields were counted and photographed under a microscope (x100).

VEGF detected by ELISA. The concentration of VEGF in the conditioned medium from human pancreatic carcinoma cells was measured using a commercially available ELISA kit (R\&D Systems, Minneapolis, MN, USA). The cells (3x10 $/$ well) were incubated overnight in 6-well dishes in medium containing $10 \%$ FBS. The media were then replaced with serum-free media containing various doses of luteolin for $24 \mathrm{~h}$. VEGF was expressed as picogram of VEGF protein $/ \mathrm{ml}$ medium and per $10^{5}$ cells. 
A

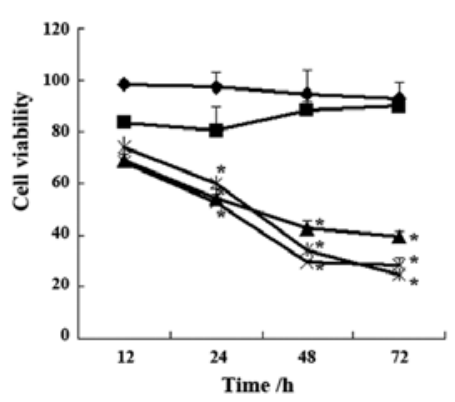

CoLo-357

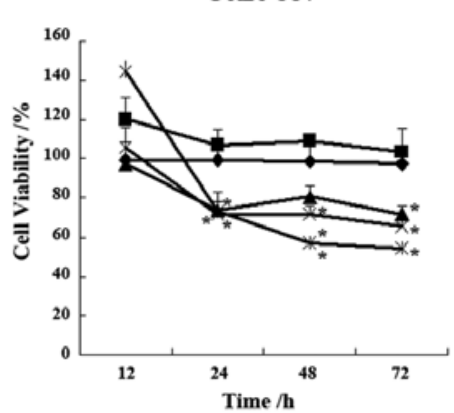

BxPC-3

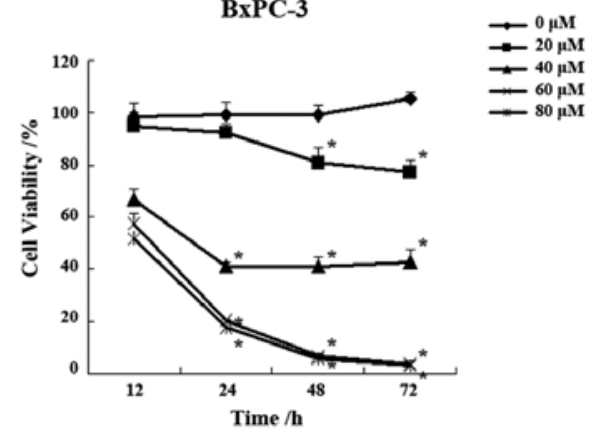

B

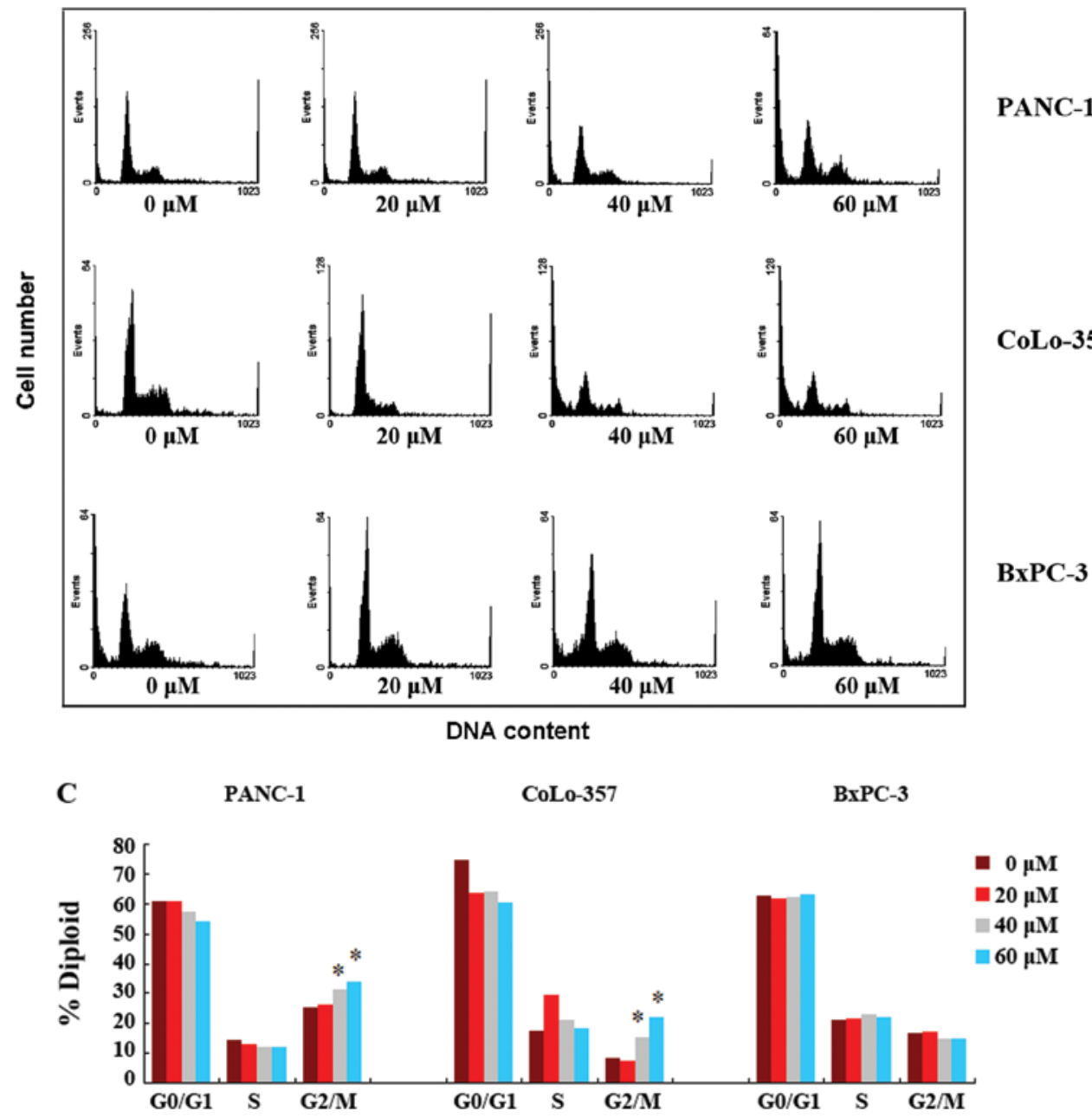

Figure 1. Cytotoxicity effect of luteolin on pancreatic carcinoma cells. (A) Inhibitory effect of luteolin on the cell proliferation of pancreatic carcinoma cells The results shown were the mean of three independent experiments for each concentration point $(20,40,60$ and $80 \mu \mathrm{M})$. (B) G2 phase cell cycle arrest by luteolin. Representative histograms depicting cell cycle distribution in pancreatic carcinoma cell cultures treated with DMSO (control) or various concentrations of luteolin for $24 \mathrm{~h}$. (C) Semi-quantification of the diploid analysis using a FACScan laser flow cytometer. Significantly different from control, ${ }^{*} \mathrm{P}<0.01$.

RT-PCR analysis. The expression of VEGF from the cell samples was studied by RT-PCR. Total cellular RNA was extracted from luteolin-treated PANC-1 cells using TRIzol reagent (Invitrogen) according to the manufacturer's instructions. Complementary DNA (cDNA) was generated with M-MLV reverse transcriptase (Takara, NY, USA) according to the manufacturer's instructions. The cDNA samples were then subjected to PCR analysis using the following primers: VEGF sense, 5'-ATGGCACCCATGGCAGAAG-3'; VEGF antisense, 5'-TCACCGCCTCGGCTTGTCAC-3'; GAPDH sense, 5'-ATGGGGAAGGTGAAGGTCG-3'; GAPDH antisense, 5'-TTACTCCTTGGAGGCCATGTG-3'. PCR reaction conditions were as follows: initial denaturation at $94^{\circ} \mathrm{C}$ for $3 \mathrm{~min}$ and 30 cycles of amplification $\left[94^{\circ} \mathrm{C}\right.$ for $45 \mathrm{sec}, 57^{\circ} \mathrm{C}$ for $45 \mathrm{sec}$ and $72^{\circ} \mathrm{C}$ for $30 \mathrm{sec}$ (GAPDH for $\left.60 \mathrm{sec}\right)$ ], followed by a final extension step for $7 \mathrm{~min}$ at $72^{\circ} \mathrm{C}$. The amplified PCR products were separated by electrophoresis on a $2 \%$ agarose gel containing ethidium bromide and quantitated by relative 
intensities of the bands as compared to those of GAPDH using Gel Base/Gel Blot/Gel Excel/Gel Sequence analysis software (UVP, UK). A value of $100 \%$ was given to the relative intensity of untreated cells (control).

$N F-\kappa B$ activation detected by EMSA. To determine NF- $\kappa \mathrm{B}$ activation by luteolin, we examined the NF- $\kappa$ B-DNA binding by electrophoretic mobility shift assay (EMSA). Briefly, nuclear extracts prepared from treated cells with Nuclear and Cytoplasmic Extraction Reagents (Pierce, IL, USA) were incubated with double-stranded $\mathrm{NF}-\kappa \mathrm{B}$ oligonucleotide (5'-AGTTGAGGGGACTTTCCCAGG-3') (Beyotime). EMSA followed the instructions of LightShift chemiluminescent EMSA kit (Pierce).

Western blot analysis. Cells were cultured until mid-log phase and then incubated with different luteolin for $24 \mathrm{~h}$. Proteins were isolated by lysis buffer (Beyotime) and measured using the Nanodrop 1000 Spectrophotometer (Thermo, IL, USA). Protein samples were separated on $13 \%$ SDS-polyacrylamide gels (SDS-PAGE) and transferred onto the PVDF membranes (Millipore). After blocked with 1\% BSA in TBST (Tris-buffered saline with Tween-20) for $2 \mathrm{~h}$, membranes were incubated with primary antibodies overnight at $4^{\circ} \mathrm{C}$. Blots were washed and incubated with secondary antibodies for $1 \mathrm{~h}$ at room temperature. Membranes were again washed three times with TBST and developed using enhanced chemiluminescence (Luminata Crescendo Western HRP substrate, Millipore). Membranes were then exposed to film.

Statistical analysis. Values were expressed as means \pm SD from three independent experiments. Statistical analysis was performed by one-way analysis of variance. When significance was detected, the t-test for multiple comparisons was performed on the data from experimental groups. A probability value of $\mathrm{P}<0.01$ was considered statistically significant.

\section{Results}

Decreased cell viability and cell cycle arrest in luteolin-treated pancreatic carcinoma cells. Exponentially growing pancreatic carcinoma cells (PANC-1, CoLo-357, BxPC-3) were cultured continuously in the absence or presence of different concentrations of luteolin. The effects of luteolin on cell growth were assessed by the commonly used MTT assay at different intervals (12, 24, 48 and $72 \mathrm{~h})$ of treatment. Luteolin treatment significantly inhibited the growth of pancreatic carcinoma cells (Fig. 1A). The degree of growth inhibition depended on both the concentration and the treatment time. At a given duration of treatment, the number of viable cells decreased as the concentration of luteolin increased. On the other hand, when luteolin concentration was held constant, the number of viable cells decreased regularly as the exposure time increased. The effect of luteolin treatment was statistically significant when compared with the control group $(\mathrm{P}<0.01)$.

To test whether luteolin could affect the cell cycle of pancreatic carcinoma cells, cells treated with DMSO or different concentration luteolin for $24 \mathrm{~h}$ were subjected to flow cytometric analysis after DNA staining. As shown in Fig. 1B and $\mathrm{C}$, exposure of PANC-1 and CoLo-357 cells to growth suppressive concentrations of luteolin resulted in a statistically significant increase in the G2 phase that was accompanied by a decrease in the G1 phase. However, luteolin had no effect on BxPC-3 cell cycle.

Luteolin-induced apoptosis in pancreatic carcinoma cells through caspase pathway. Differences in cell morphology were observed between luteolin-treated and control cells by light microscopy. The most conspicuous change observed in luteolin-treated cells included cell shrinkage and extensive detachment of the cells from the cell culture substratum (Fig. 2A). These changes, which were characteristic of cell apoptotic death, became visible after luteolin treatment, but were absent in control cells. The morphological change became more remarkable with increased time of drug treatment (data not shown). The occurrence of apoptosis was further verified by Hoechst staining, which detects chromatin condensation, one of the hallmarks of apoptotic cell death. Differences were observed in the nuclei of luteolin-treated and untreated pancreatic carcinoma cells after staining with Hoechst 33258 (Fig. 2A). The Hoechst 33258 dye stained morphologically normal nuclei dimly blue, whereas luteolin-treated cells demonstrated smaller nuclei with brilliant blue staining. The change in nuclear morphology was initially observed after $24 \mathrm{~h}$ of luteolin treatment and increased thereafter (data not shown). These results demonstrated that luteolin induces morphological changes characteristic of cell apoptotic death.

The molecular mechanism for the potent pro-apoptotic effect of luteolin on pancreatic carcinoma cells was further studied. Western blot analysis was done as described in Materials and methods. As shown in Fig. 2B, luteolin increased the expression of pro-apoptotic protein (Bax) and decreased anti-apoptotic protein (Bcl-2), with a concomitant increase in the levels of caspase-3 and cleaved PARP in pancreatic carcinoma cells after treatment for $24 \mathrm{~h}$.

Differential growth inhibition of luteolin on HUVEC and $P B M C$. In order to rule out the effects of physico-chemical property of luteolin, such as $\mathrm{pH}$, but not the pharmacologic actions on the cells, human normal cells PMBC were used as control. The inhibition effect of luteolin on cell growth was assessed by the commonly used MTT assay. Luteolin treatment significantly inhibited the growth of HUVEC cells in concentration-dependent manner (Fig. 3A) with $\mathrm{IC}_{50}$ value of $47.0 \mu \mathrm{M}$. However, no significant inhibitory effect was observed on PBMC.

Luteolin inhibits angiogenesis in vivo. Anti-angiogenic activity of luteolin on CAM was assayed. The CAM of the chicken embryo provides a unique model for investigating the process of new blood vessel formation and vessel responses to anti-angiogenic agents. Using this model, we additionally examined the potential in vivo anti-angiogenic activity of luteolin. New blood vessels formed well on CAM in the control group. Luteolin at $5 \mathrm{nmol} / \mathrm{egg}$ incubation for $72 \mathrm{~h}$ showed a notable restraint. Up to $10 \mathrm{nmol} / \mathrm{egg}$, the inhibition was getting more prominent (Fig. 3B). These results demonstrate that luteolin was able to suppress angiogenesis in embryos.

Co-culture of HUVEC with PANC-1 activated HUVEC tube formation, which were suppressed by luteolin. To investigate 
$\mathbf{A}$
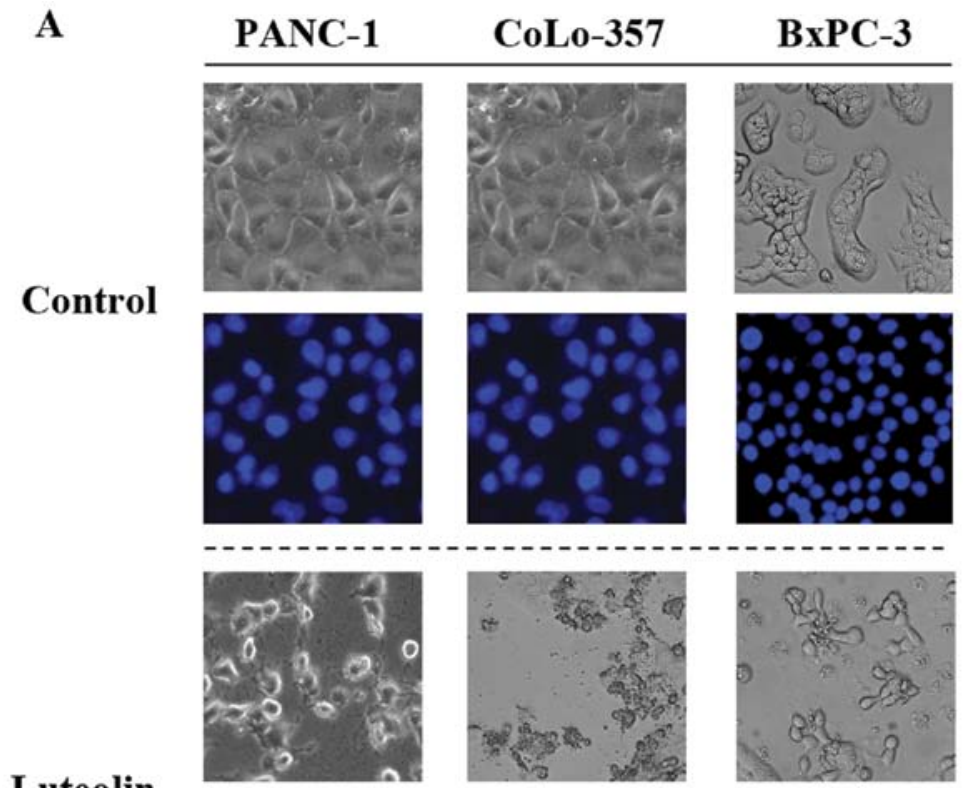

Luteolin
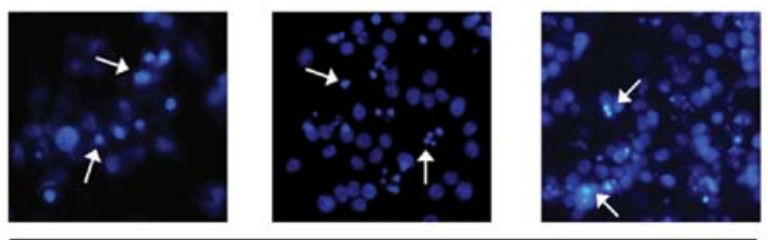

B

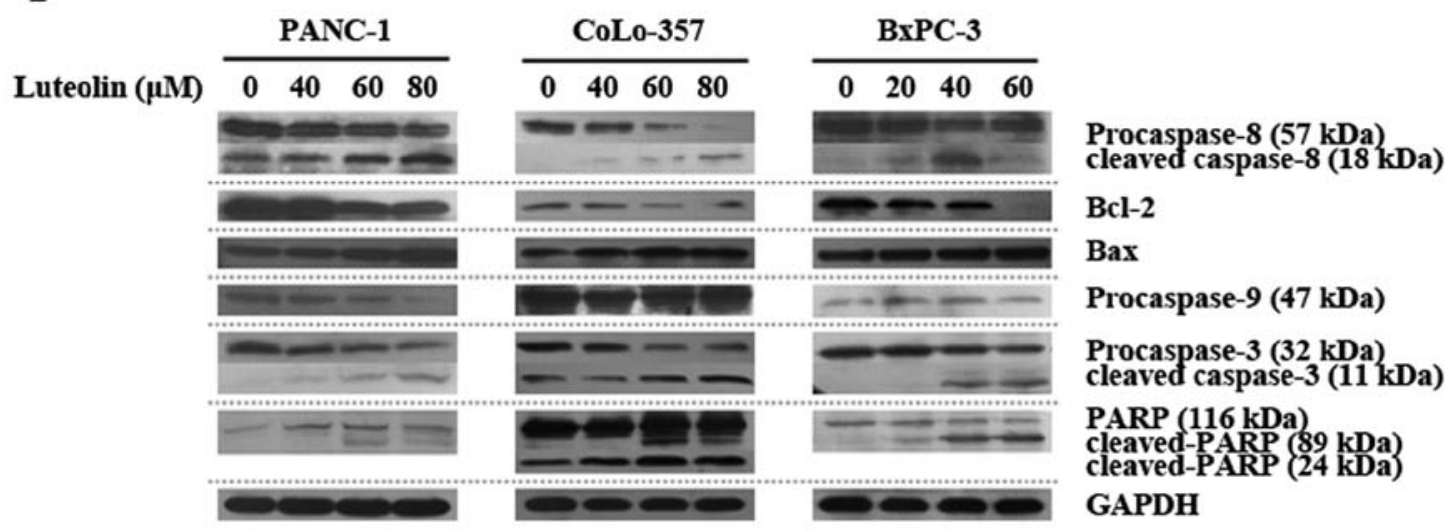

Figure 2. Luteolin-induced apoptosis of pancreatic carcinoma cells. (A) Nuclear staining of pancreatic carcinoma cells with Hoechst 33258. Compared to control (DMSO), $40 \mu \mathrm{M}$ luteolin-treated cells demonstrated smaller nuclei with brilliant blue staining (white arrows). (B) Western blot assay of apoptosis related protein.

the effects of PANC-1 cells on HUVEC angiogenesis, we used Transwell inserts with a pore size of $0.4 \mathrm{~mm}$ and no collagen coating. HUVEC were cultured in the chamber coated by Matrigel with PANC-1 cells in the inserts. When cultured alone in the growth factor-reduced Matrigel, HUVEC barely formed capillary structures $12 \mathrm{~h}$ after plating. However, HUVEC co-cultured with PANC-1 cells displayed a dramatically increased network formation and this effect disappeared when PANC-1 cells pre-treated with $40 \mu \mathrm{mol} / 1$ luteolin for $30 \mathrm{~min}$ (Fig. 3C). These data suggested that pancreatic carcinoma cells can induce endothelial cells to differentiate into structures that resemble in vivo neovascularization and this effect could be inhibited by luteolin.

Luteolin suppresses VEGF secretion from pancreatic carcinoma cells. It has been shown that VEGF, actively secreted from hypoxic tumor cells, could potently trigger tumor angiogenesis. Reduction of VEGF weakens its stimulation for tumor angiogenesis (7). We additionally examined the effect of luteolin on VEGF secretion from the pancreatic carcinoma cells by ELISA analysis. The results showed that luteolin treatment for $24 \mathrm{~h}$ decreased VEGF secretion compared to the vehicle control group (Fig. 4A). Luteolin ( $40 \mu \mathrm{mol} / \mathrm{l})$ decreased VEGF secretion by $83.1 \%$ (PANC-1 cells), $51.4 \%$ (CoLo-357 cells) and $55.9 \%$ (BxPC-3 cells).

Luteolin suppresses production of VEGF $m R N A$. The expression of intracellular VEGF protein was detected by western blotting after treating with luteolin for $24 \mathrm{~h}$. As shown in Fig. 4B, VEGF protein in untreated or treated-PANC-1 cells had no significant difference. Further, we detected the VEGF mRNA level by RT-PCR. Gene expression of these two growth 
$\mathbf{A}$

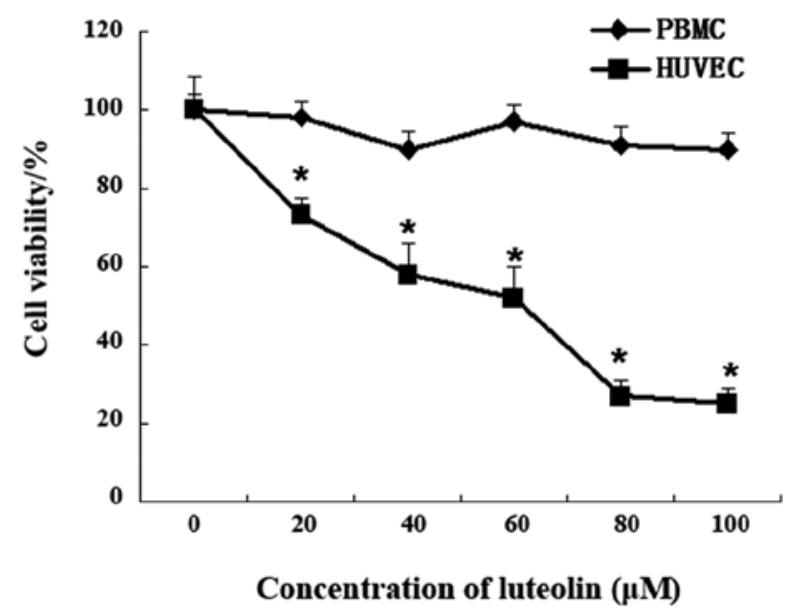

B

$0 \mathrm{nmol} / \mathrm{egg}$

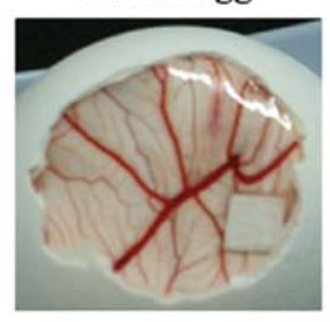

5 nmol/egg

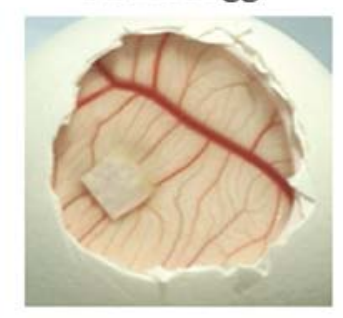

$10 \mathrm{nmol} / \mathrm{egg}$

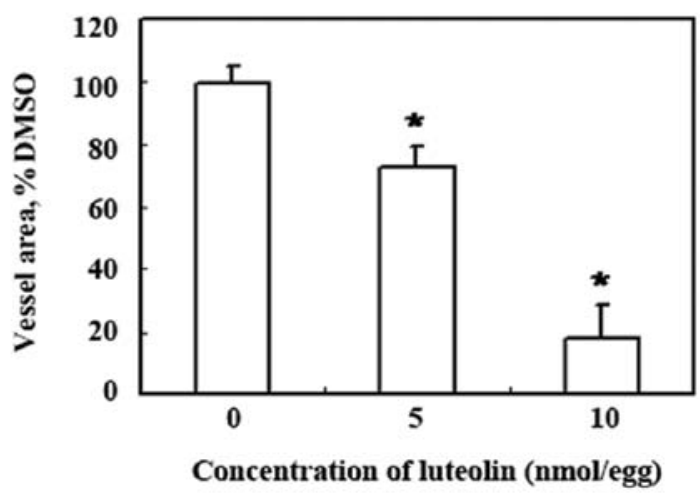

C
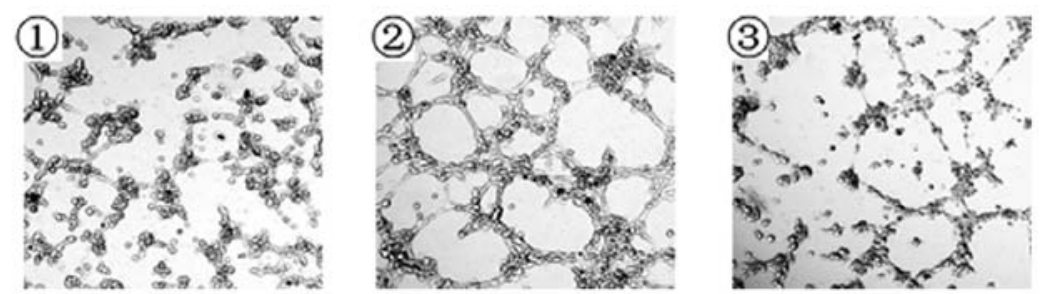

Figure 3. (A) Proliferation inhibition effect of luteolin on HUVEC and PBMC. (B) Luteolin inhibits angiogenesis in vivo. Photographs are representative from five independent experiments. (C) Effect of the PANC-1 cells on HUVEC network formation in the co-culture systems. 1, HUVEC cultured alone for 12 h. 2, PANC-1 cells and HUVEC co-cultured for $12 \mathrm{~h}$. Marked elongated HUVEC network formation was observed. 3 , PANC-1 cells pre-treated with $40 \mu$ mol/1 luteolin for $30 \mathrm{~min}$, then co-cultured with HUVEC for $12 \mathrm{~h}$. (x 100).

factors were both decreased compared to the vehicle control group (Fig. 4C). These data suggested that the expression level of VEGF mRNA was decreased after treatment with luteolin, but not the intracellular VEGF protein level.

Luteolin suppresses VEGF secretion related to JNK phosphorylation and $N F-\kappa B-D N A$ binding activity. Our results showed that luteolin suppressed VEGF secretion from pancreatic carcinoma cells and antagonized VEGF-indued angiogenesis in HUVEC. This prompted us to investigete how luteolin regulate the secretion of VEGF. Since the secretion of VEGF was regulated by MAP kinase pathways (8), we examined the expression and phosphorylation of MAPK (ERK1/2, p38 and JNK). Western blotting result showed that the expression and phosphorylation of ERK1/2 and p38 had no significant difference compared with the control group (Fig. 4D). However, 

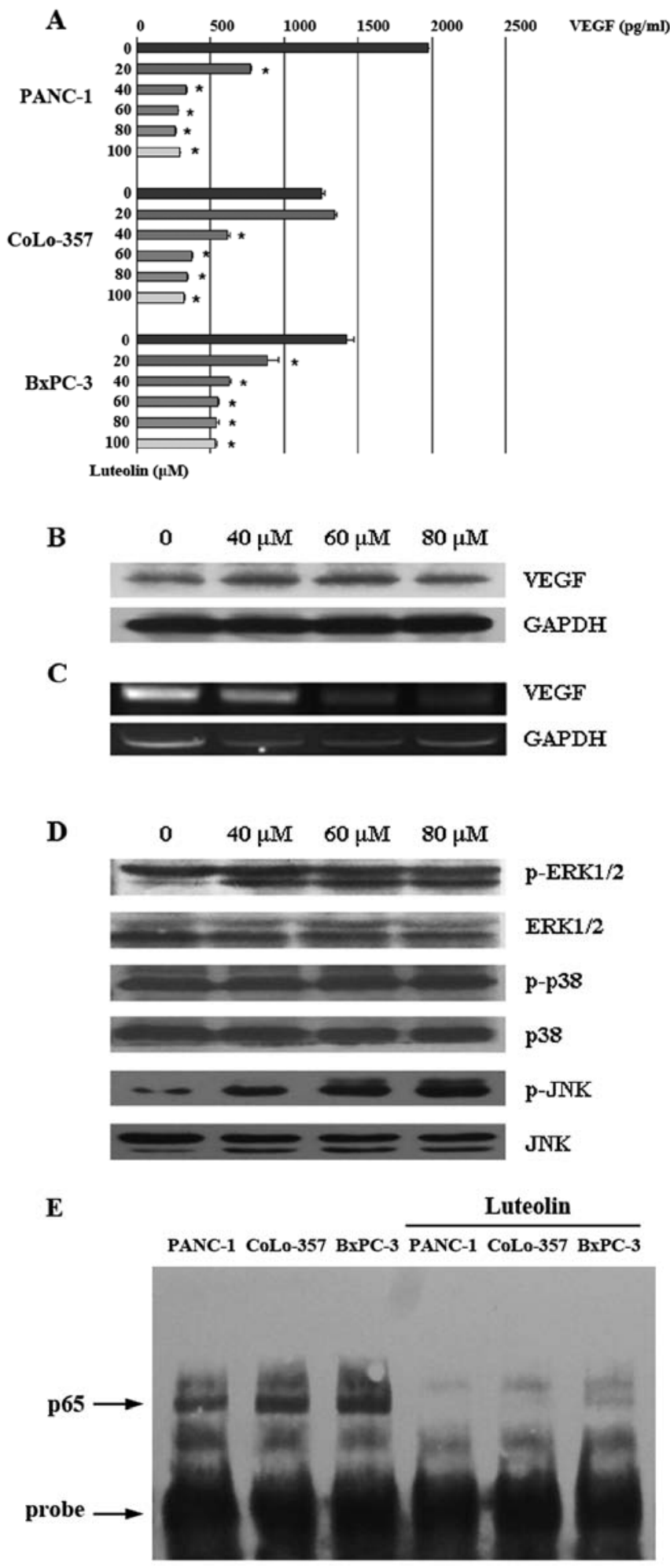

Figure 4. (A) Luteolin suppresses VEGF secretion in pancreatic carcinoma cells. Representative data are shown from three independent experiments with identical results. Significantly different from control, ${ }^{*} \mathrm{P}<0.01$. (B and C) Luteolin inhibited the mRNA expression level (C) but not the VEGF protein level (B) in PANC-1 cells. (D) Western blot analysis of MAPK pathway. Total cell lysates of PANC-1 cells treated with or without luteolin for the indicated time were analyzed by SDS-PAGE and, subsequently, immunoblotted with antibodies against p-ERK1/2, ERK1/2, p-p38, p38, JNK1/2/3, p-JNK1/2/3 and GAPDH. (E) Luteolin inhibits NF- $\mathrm{kB}$ transcription activity on pancreatic carcinoma cells. Pancreatic carcinoma cells were incubated with luteolin (60 $\mu \mathrm{M}$ for PANC-1 and CoLo-357 cells, $40 \mu \mathrm{M}$ for BxPC-3 cells) for $7 \mathrm{~h}$ at $37^{\circ} \mathrm{C}$. DMSO $(0.1 \%)$ was used as control. Nuclear extracts were prepared and then tested for NF- $\mathrm{kB}$ activity by electrophoretic mobility shift assay as described in Materials and methods.

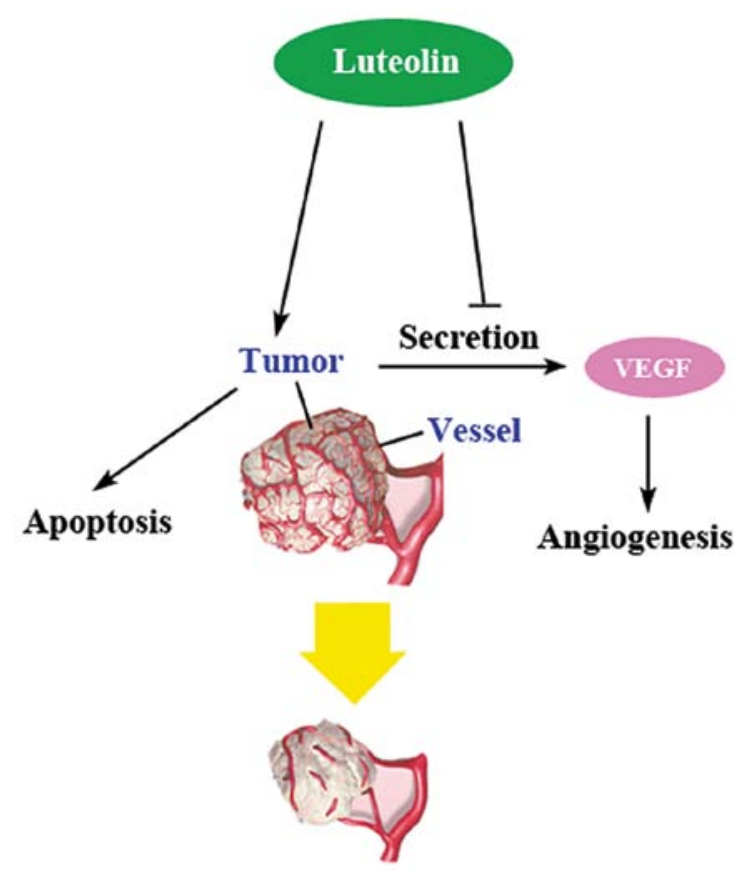

Figure 5. A mechanism of luteolin-induced apoptosis in pancreatic carcinoma cells. Anti-angiogenesis was generated in luteolin-treated cells undergoing apoptosis.

phosphorylation level of JNK was increased, which could induce apoptosis (6). These data suggested that the secretion of VEGF may be regulate by JNK signaling pathway. The result from RT-PCR revealed the VEGF mRNA level was decreased in a concentration-dependent manner after luteolin treatment (Fig. 4C). Therefore, we hypothesized that the abatement of VEGF secretion was due to inhibition of VEGF mRNA expression. Our recent studies showed that luteolin inhibited TNF $\alpha$ induced NF- $\kappa$ B translocation on A549 cells (6). VEGF mRNA expression was partly regulated by nuclear transcription factor NF- $\kappa \mathrm{B}$. In view of the above, EMSA assay was done to investigate the NF- $\mathrm{B}-\mathrm{DNA}$ binding activity. The result showed that luteolin inhibited $\mathrm{NF}-\kappa \mathrm{B}$ transcription activity in all three pancreatic carcinoma cells (Fig. 4E).

\section{Discussion}

Angiogenesis is the physiological process involving the growth of new blood vessels from pre-existing vessels (9). Angiogenesis is a necessary and required step for transition from a small harmless cluster of cells to a large tumor. Tumors cannot grow beyond a certain size, generally $1-2 \mathrm{~mm}^{3}$ (10), due to a lack of oxygen and other essential nutrients. Angiogenesis is also required for the spread of a tumor or metastasis. Luteolin was found to be a potent angiogenesis inhibitor (11). In a murine xenograft tumor model, luteolin inhibited tumor growth and angiogenesis in xenografted tumors (12). In this study, we investigated the effect of luteolin on angiogenesis in vitro with human umbilical vein endothelial cells. Luteolin showed significant inhibitory to HUVEC, but had no effect on human normal cell PBMC. In vivo experiment, luteolin at $5 \mathrm{nmol} / \mathrm{egg}$ incubation for $72 \mathrm{~h}$ showed a notable inhibition of new blood vessel formation in 
CAM. These result indicate that luteolin may be used as an anti-angiogenesis agent with little side effect.

The significant role of vegetables and fruits in prevention and treatment of cancer is due to their high polyphenol content, particularly of flavonoids (13). Luteolin is a common dietary flavonoid that can be found in a large quantity of plants and foods. Luteolin was reported to inhibit the development of a series of solid tumors (colonic HT-29, HCT116, hepatic HepG2, SK-Hep-1, PLC/PRF/5, Hep3, cervical HeLa, oral SCC-4) (14-23), but the mechanism of luteolin affecting pancreatic carcinoma cells was not addressed. Therefore, we used three different human pancreatic carcinoma cell lines, the poorly differentiated cell line (PANC-1), the moderately differentiated cell line (CoLo-357) and the well differentiated cell line (BxPC-3) as model to examine the therapeutic effects of luteolin. In our studies, luteolin inhibited the proliferation of those three pancreatic carcinoma cell lines and induced apoptotic cell death through caspase pathway. Luteolin induced G2 phase arrest of cell cycle progression on PANC-1 and CoLo-357 cells. Since pancreatic carcinoma shows strong tumor angiogenesis, overexpression of VEGF, we detected the VEGF expression in pancreatic carcinoma cells with western blotting and VEGF secreted into the medium with ELISA. The results showed that intracellular VEGF protein expression level was constant, when the VEGF secreted into the medium decreased significantly. This prompted us to investigate whether the inhibition of VEGF secretion on pancreatic carcinoma cells could be anti-angiogenic. Therefore, we co-cultured the luteolin-treated PANC-1 cells with HUVEC. As speculated, the capillary-like structures, consisting of HUVEC, decreased compared with the luteolin-untreated group.

Since the secretion of VEGF was regulated by the MAP kinase pathway, three key MAP kinases (ERK1/2, p38 and JNK) were chosen for investigation. The phosphorylation of JNK was upregulated by luteolin, when p-ERK1/2 and p-p38 did not change. Substantial research on luteolin has revealed that luteolin could either activate JNK $(15,24)$ or downregulate JNK phosphorylation (25-27), both leading to apoptosis. These data suggested that the secretion of VEGF could be regulated by the JNK signaling pathway. The result from RT-PCR revealed the VEGF mRNA level was decreased in a concentration-dependent manner after luteolin treatment. Therefore, we hypothesized that the abatement of VEGF secretion was due to inhibition of VEGF mRNA expression. VEGF mRNA expression was partly regulated by nuclear transcription factor $\mathrm{NF}-\kappa \mathrm{B}$. EMSA assay showed that luteolin inhibited $\mathrm{NF}-\kappa \mathrm{B}$ transcription activity in all three pancreatic carcinoma cells. How luteolin affects the NF- $\mathrm{BB}$ transcription activity is now under study in our laboratory.

Taking these results together, we demonstrated that luteolin possessed dual roles, the direct action on endothelial cells and reduction of the VEGF secretion from tumor cells (Fig. 5). Such dual activities of luteolin, different from the relatively single effects of other angiogenesis inhibitors, can be reasonably inferred to allow better in vivo and even better clinical effects as a novel antitumor agent.

\section{Acknowledgements}

This study was supported by the National Natural Science Foundation of China (Nos. 30701098,30873410 and 81073101),
Natural Science Foundation of Zhejiang Province (No. Y2090676) and Jiangsu Province's Outstanding Leader Program of Traditional Chinese Medicine.

\section{References}

1. Neesse A, Gress TM and Michl P: Therapeutic targeting of apoptotic pathways: novel aspects in pancreatic cancer. Curr Pharm Biotechnol: May 24, 2011 (Epub ahead of print).

2. Kato H, Ishikura H, Kawarada Y, Furuya M, Kondo S and Yoshiki T: Anti-angiogenic treatment for peritoneal dissemination of pancreas adenocarcinoma: a study using TNP-470. Jpn J Cancer Res 92: 67-73, 2001

3. Saif MW: Anti-angiogenesis therapy in pancreatic carcinoma. JOP 7: 163-173, 2006

4. Lin Y, Shi R, Wang X and Shen HM: Luteolin, a flavonoid with potential for cancer prevention and therapy. Curr Cancer Drug Targets 8: 634-646, 2008.

5. Jaffe EA, Nachman RL, Becker CG and Minick CR: Culture of human endothelial cells derived from umbilical veins. Identification by morphologic and immunologic criteria. J Clin Invest 52: 2745-2756, 1973.

6. Cai X, Ye T, Liu C, et al: Luteolin induced G2 phase cell cycle arrest and apoptosis on non-small cell lung cancer cells. Toxicol In Vitro 25: 1385-1391, 2011.

7. Minchenko A, Bauer T, Salceda S and Caro J: Hypoxic stimulation of vascular endothelial growth factor expression in vitro and in vivo. Lab Invest 71: 374-379, 1994.

8. Nagineni CN, Samuel W, Nagineni S, et al: Transforming growth factor- $\beta$ induces expression of vascular endothelial growth factor in human retinal pigment epithelial cells: involvement of mitogen-activated protein kinases. J Cell Physiol 197: 453-462, 2003.

9. Das A and McGuire PG: Retinal and choroidal angiogenesis: pathophysiology and strategies for inhibition. Prog Retin Eye Res 22: 721-748, 2003.

10. McDougall SR, Anderson AR and Chaplain MA: Mathematical modelling of dynamic adaptive tumour-induced angiogenesis: clinical implications and therapeutic targeting strategies. J Theor Biol 241: 564-589, 2006.

11. Joussen AM, Rohrschneider K, Reichling J, Kirchhof B and Kruse FE: Treatment of corneal neovascularization with dietary isoflavonoids and flavonoids. Exp Eye Res 71: 483-487, 2000.

12. Bagli E, Stefaniotou M, Morbidelli L, et al: Luteolin inhibits vascular endothelial growth factor-induced angiogenesis; inhibition of endothelial cell survival and proliferation by targeting phosphatidylinositol 3'-kinase activity. Cancer Res 64: 7936-7946, 2004.

13. Ramos S: Effects of dietary flavonoids on apoptotic pathways related to cancer chemoprevention. J Nutr Biochem 18: 427-442, 2007.

14. Chang J, Hsu Y, Kuo P, Kuo Y, Chiang L and Lin C: Increase of $\mathrm{Bax} / \mathrm{Bcl}-\mathrm{XL}$ ratio and arrest of cell cycle by luteolin in immortalized human hepatoma cell line. Life Sci 76: 1883-1893, 2005.

15. Lee HJ, Wang CJ, Kuo HC, Chou FP, Jean LF and Tseng TH: Induction apoptosis of luteolin in human hepatoma HepG2 cells involving mitochondria translocation of $\mathrm{Bax} / \mathrm{Bak}$ and activation of JNK. Toxicol Appl Pharmacol 203: 124-131, 2005.

16. Lee WJ, Wu LF, Chen WK, Wang CJ and Tseng TH: Inhibitory effect of luteolin on hepatocyte growth factor/scatter factorinduced HepG2 cell invasion involving both MAPK/ERKs and PI3K-Akt pathways. Chem Biol Interact 160: 123-133, 2006.

17. Lim do Y, Jeong Y, Tyner AL and Park JH: Induction of cell cycle arrest and apoptosis in HT-29 human colon cancer cells by the dietary compound luteolin. Am J Physiol Gastrointest Liver Physiol 292: G66-G75, 2007.

18. Plaumann B, Fritsche M, Rimpler H, Brandner G and Hess RD: Flavonoids activate wild-type p53. Oncogene 13: 1605-1614, 1996.

19. Zhang Q, Zhao XH and Wang ZJ: Flavones and flavonols exert cytotoxic effects on a human oesophageal adenocarcinoma cell line (OE33) by causing $\mathrm{G} 2 / \mathrm{M}$ arrest and inducing apoptosis. Food Chem Toxicol 46: 2042-2053, 2008.

20. Schutte ME, Boersma MG, Verhallen DA, Groten JP and Rietjens IM: Effects of flavonoid mixtures on the transport of 2-amino-1-methyl-6-phenylimidazo[4,5-b]pyridine (PhIP) through Caco-2 monolayers: an in vitro and kinetic modeling approach to predict the combined effects on transporter inhibition. Food Chem Toxicol 46: 557-566, 2008. 
21. Xavier CP, Lima CF, Preto A, Seruca R, Fernandes-Ferreira M and Pereira-Wilson C: Luteolin, quercetin and ursolic acid are potent inhibitors of proliferation and inducers of apoptosis in both KRAS and BRAF mutated human colorectal cancer cells. Cancer Lett 281: 162-170, 2009.

22. Yang SF, Yang WE, Chang HR, Chu SC and Hsieh YS: Luteolin induces apoptosis in oral squamous cancer cells. J Dent Res 87: 401-406, 2008

23. Yee SB, Lee JH, Chung HY, et al: Inhibitory effects of luteolin isolated from Ixeris sonchifolia Hance on the proliferation of HepG2 human hepatocellular carcinoma cells. Arch Pharm Res 26: $151-156,2003$

24. Shi RX, Ong CN and Shen HM: Luteolin sensitizes tumor necrosis factor-alpha-induced apoptosis in human tumor cells. Oncogene 23: 7712-7721, 2004.
25. Ando C, Takahashi N, Hirai S, et al: Luteolin, a food-derived flavonoid, suppresses adipocyte-dependent activation of macrophages by inhibiting JNK activation. FEBS Lett 583: 3649-3654, 2009.

26. Jang S, Kelley KW and Johnson RW: Luteolin reduces IL-6 production in microglia by inhibiting JNK phosphorylation and activation of AP-1. Proc Natl Acad Sci USA 105: 7534-7539, 2008.

27. Kimata M, Shichijo M, Miura T, Serizawa I, Inagaki $N$ and Nagai H: Effects of luteolin, quercetin and baicalein on immunoglobulin E-mediated mediator release from human cultured mast cells. Clin Exp Allergy 30: 501-508, 2000. 